\title{
Monitoramento terapêutico de antibióticos utilizando dbs - dried blood spots (mancha de sangue seco em papel): uma facilidade na prática clínica
}

\author{
Letícia Vale Scribel \\ Instituto Federal de Educação, Ciência e Tecnologia do Rio Grande do Sul (IFRS) \\ Campus Porto Alegre \\ (leticia.scribel@poa.ifrs.edu.br) \\ Nadine Bordin Andriguetti \\ Universidade Feevale - Novo Hamburgo \\ (nadineba@live.com) \\ Rafael Linden \\ Universidade Feevale - Novo Hamburgo \\ (rafael.linden@feevale.br)
}

Alexandre Zavascki

Universidade Federal do Rio Grande do Sul (UFRGS)

(azavascki@hcpa.edu.br)

Resumo: A maior preocupação atual relacionada aos antimicrobianos é a resistência bacteriana. Enquanto não surgem novos antibióticos no mercado, o monitoramento terapêutico (MT) é essencial para individualização da terapia antibacteriana e prevenção do surgimento de cepas resistentes. $O$ MT depende do acompanhamento da concentração sanguínea do medicamento, que pode ser obtida através de sangue venoso ou sangue capilar. A coleta de sangue capilar obtida por punção digital é chamada de DBS (do inglês Dried Blood Spots, ou mancha de sangue seco em papel) e apresenta vantagens em relação à coleta usual por punção venosa: é menos invasiva, mais estável e requer menor volume de sangue. Assim, permite a realização do MT em pacientes de difícil acesso venoso como idosos, neonatos, bebês e crianças, facilita a rotina de coleta da equipe assistencial e permite a auto coleta. Por fornecer pequenas quantidades de sangue (aproximadamente $50 \mu \mathrm{L}$ ), amostras em DBS são analisadas por métodos altamente sensíveis e precisos como Cromatografia Líquida acoplada à Espectrometria de Massas em "tandem" (LC-MS/MS).

Palavras-chave: Monitoramento terapêutico; Antibióticos; Dried Blood Spots; Cromatografia Líquida acoplada à Espectrometria de Massas. 


\title{
Therapeutic monitoring of antibiotics using dried blood spots: a facility in clin- ical practice
}

\begin{abstract}
The major current concern regarding antimicrobials is bacterial resistance. While no new antibiotics are on the market, therapeutic monitoring (TM) is essential for antibacterial therapy individualization and prevention of emergence of resistant strains. The MT depends on drug blood concentration monitoring, which can be obtained through venous blood or capillary blood. Capillary blood collection by digital puncture is called DBS (Dried Blood Spots) and has advantages over the usual collection by venipuncture: it is less invasive, more stable and requires less volume of blood. Thus, it allows the accomplishment of TM in patients with difficult venous access such as the elderly, neonates, infants and children, facilitates the care staff routine of collection and allows self-collection. As DBS provides small amounts of blood (approximately $50 \mu \mathrm{L}$ ), DBS samples are analyzed by the Liquid Chromatography method coupled with Tandem Mass Spectrometry (LC-MS / MS), which provides results with sensitivity and precision.
\end{abstract}

Keywords: Therapeutic drug monitoring; Antibiotics; Dried Blood Spots; Liquid chromatography coupled with tandem mass spectrometry.

\section{INTRODUÇÃO}

A resistência microbiana é um dos principais destaques da OMS (Organização Mundial da Saúde). Segundo a OMS, a resistência aos antibióticos é uma das maiores ameaças à saúde global. Pode afetar qualquer pessoa, de qualquer idade em qualquer lugar. Ocorre naturalmente, mas o uso irracional dos antibióticos em humanos e animais está acelerando o processo. Diversas infecções estão se tornando difíceis de tratar, como pneumonia, tuberculose e gonorreia, pois os antibióticos usados estão se tornando menos efetivos. A resistência bacteriana leva ao aumento no tempo de internação, elevação nos custos do tratamento e aumento da mortalidade (OMS, 2018).

O Monitoramento Terapêutico (MT) é uma especialidade clínica multidisciplinar utilizada para otimizar e individualizar a terapia medicamentosa na população em geral ou em populações especiais (MILOSHESKA et al., 2015). Especificamente para os antimicrobianos, o MT tem papel importante na redução ou prevenção de resistência microbiana, bem como na otimização da dosagem de antibióticos já existentes, considerando a falta de alternativa terapêutica em casos de infecções por microrganismos multirresistentes. Para alguns medicamentos, o objetivo é alcançado ao minimizar efeitos adversos ou está relacionado à toxicidade, enquanto que para outros os benefícios são alcançados ao atingir efeitos 
terapêuticos desejados especialmente em doses não usuais (PAGE-SHARP et al., 2015; BARLAM et al., 2016).

A individualização da terapia é baseada no perfil farmacocinético (PK) e farmacodinâmico (PD) do paciente, que depende do conhecimento da concentração sanguínea do medicamento após sua administração (RYBAK et al., 2009; KATO et al., 2017). A dosagem do nível sérico dos medicamentos pode ser realizada utilizando sangue venoso ou capilar. A coleta de sangue capilar é realizada por DBS (do inglês Dried Blood Spots, ou mancha de sangue seco em papel) e apresenta vantagens em relação à coleta usual por punção venosa por ser menos invasiva, mais estável e por requerer menor volume de sangue. Assim, permite a realização do MT em pacientes especiais, como neonatos, bebês e crianças, facilita a rotina de coleta da equipe assistencial e permite a auto coleta. Uma vez que oferece menor quantidade de amostra, é necessária análise laboratorial mais sensível, facilmente obtida através do método de Cromatografia Líquida acoplada à Espectrometria de Massas em "tandem" (LC-MS/MS) (KEEVIL, 2011; ANTUNES et al., 2016; MAHMOUDI et al., 2014).

Esta revisão da literatura visa apresentar conceitos relacionados ao MT de antibióticos, ao uso da coleta de sangue capilar por DBS e aos métodos utilizados para dosagem das concentrações sanguíneas.

\section{MÉTODOS}

Foi realizada uma revisão narrativa da literatura. A estratégia de busca envolveu as seguintes bases de dados: LILACS, SciELO, PubMed e banco de teses da CAPES, priorizando as referências mais recentes. Foram realizadas buscas através dos termos "therapeutic drug monitoring", "DBS", "Dried Blood Spots", "liquid chromatography coupled with tandem mass spectrometry", "LC-MS/MS", "haematocrit" e suas combinações. Foram consultadas, também, algumas referências citadas nos artigos encontrados através da busca nas bases de dados relacionadas. 


\section{RESULTADOS}

A quantidade de publicações encontradas e utilizadas nesta revisão está relacionada de acordo com as bases de dados consultadas conforme a Figura 1.

Figura 1 - Estratégia de busca de referências bibliográficas que contemplam os temas deste estudo e os resultados em número de artigos obtidos. A coluna central indica as palavraschave e suas combinações, representadas pelos números de 1 a 6 conforme a legenda. As colunas coloridas indicam a quantidade de artigos obtidos de acordo com o site consultado.

As colunas mais externas indicam os artigos consultados nesta revisão.

2- Dried Blood Spots
3- liquid
chromatography
mass spectrometry
4- amikacin
6- vancomycin
9- haematocrit

\section{DISCUSSÃO}

\section{Monitoramento Terapêutico}

O objetivo principal do MT é a otimização da terapia individualizada. Para alguns medicamentos, este objetivo é alcançado ao minimizar a efeitos adversos ou estão relacionados à toxicidade, enquanto que para outros os benefícios são alcançados ao atingir efeitos terapêuticos desejados especialmente em doses não 
usuais. Aqueles que frequentemente produzem toxicidade em doses próximas às indicadas são usualmente monitorados. Sendo o intervalo da concentração sérica alvo estreito, há necessidade da seleção precisa da dosagem do medicamento, bem como seu intervalo de administração. O intervalo terapêutico é a faixa da concentração onde a probabilidade da resposta clínica desejada é relativamente alta e a probabilidade de apresentar toxicidade é relativamente baixa (BURTON et al., 2006).

Estudos recentes sugerem que as doses usuais de antibióticos não atingem os melhores resultados em pacientes críticos devido à sua variabilidade farmacocinética e sua farmacodinâmica alterada. A adaptação da dose é desafiadora devido à imprevisível relação dose-exposição. Consequentemente, defende-se a individualização da dosagem do antibiótico. As doses convencionais de muitos antibióticos frequentemente resultam em exposição abaixo ou acima da terapêutica desejada. A otimização da dosagem baseada em princípios PK/PD podem efetivamente melhorar a exposição ao antibiótico. Assim, o MT é a abordagem mais indicada a fim de otimizar e individualizar sua dosagem (PARKER et al., 2015).

\section{Princípios farmacocinéticos e farmacodinâmicos}

O MT é baseado em princípios farmacocinéticos e farmacodinâmicos PK/PD, fazendo-se necessário monitorar a concentração sérica do medicamento em questão (BURTON et al., 2006). O perfil farmacocinético refere-se à concentração de um medicamento no sangue ou nos tecidos relacionada ao tempo, refletindo sua absorção, distribuição, biotransformação e eliminação, ou seja, o caminho que ele faz no organismo. O perfil farmacodinâmico diz respeito às ações farmacológicas e aos mecanismos pelos quais os fármacos atuam, ou seja, os efeitos que os fármacos provocam no organismo. A abordagem PK/PD explora a relação triangular entre a concentração sanguínea do antimicrobiano, a sensibilidade do microrganismo ao antibiótico (medida pela Concentração Inibitória Mínima - CIM) e desfechos clínicos pré-definidos (KATO et al., 2017; PAGE-SHARP et al., 2015; RYBAK et al., 2009).

O perfil farmacocinético é importante para calcular a dose apropriada e a frequência de administração, para ajustar doses nos pacientes e para fazer 
comparações entre os vários fármacos. Assim, faz-se necessário monitorar a concentração sanguínea do medicamento em questão. Por questões de factibilidade - custo, disponibilidade de pessoal treinado, adesão da equipe assistencial e do paciente -, não são todos os medicamentos que são monitorados na rotina hospitalar através do perfil farmacocinético individualizado. Assim, são selecionados aqueles que frequentemente produzem toxicidade em doses próximas às indicadas, por exemplo (BURTON et al., 2006). Neste caso, sendo o intervalo da concentração sérica alvo estreito, há necessidade do cálculo mais preciso da dosagem do medicamento, bem como seu intervalo de administração. O intervalo terapêutico é a faixa da concentração onde a probabilidade da resposta clínica desejada é relativamente alta e a probabilidade de apresentar toxicidade é relativamente baixa (BURTON et al., 2006).

Estudos recentes (PARKER et al., 2015) sugerem que as doses usuais de antibióticos não atingem os melhores resultados em pacientes críticos devido à sua variabilidade farmacocinética e sua farmacodinâmica alterada. A adaptação da dose é desafiadora devido à imprevisível relação dose-exposição. Consequentemente, defende-se individualizar a dosagem do antibiótico. As doses convencionais de muitos antibióticos frequentemente resultam em exposição abaixo ou acima da terapêutica desejada. A otimização da dosagem baseada em princípios PK/PD podem efetivamente melhorar a exposição ao antibiótico (PARKER et al., 2015).

\section{Métodos de Coleta de Sangue}

A análise de medicamentos é normalmente realizada em amostras de soro ou plasma, obtidos por punção venosa. Entretanto, há um crescente interesse em sangue capilar obtido por punção digital utilizando-se uma lanceta automática, colhido em uma matriz de papel filtro, chamada de DBS. Comparada com a coleta convencional de sangue venoso, a coleta em DBS é simples e conveniente proporcionando melhor conforto para o paciente (WILHELM et al., 2014). Além disso, a dosagem da concentração sanguínea de medicamentos depende da disponibilidade de metodologias laboratoriais sofisticadas, disponíveis apenas em centros especializados, com acesso limitado à maioria dos pacientes. Neste contexto, o interesse no uso de DBS vem aumentando, permitindo uma logística simples, estável e barata (ANTUNES et al., 2016). 
As vantagens potenciais do uso de sangue capilar são a facilidade de coleta, que eventualmente poderá ser realizada pelo próprio paciente e que poderá ser feita fora do ambiente hospitalar, ou facilitada na ausência de acesso venoso em pacientes internados. Por ser um processo menos invasivo e menos doloroso, é mais aceitável principalmente por crianças e também torna mais rápida e efetiva a coleta da amostra (KEEVIL, 2011; ANTUNES et al., 2016). Outras vantagens do uso de DBS são a facilidade no transporte, o armazenamento e a maior estabilidade, permitindo a obtenção de amostras em situações onde a coleta de sangue ou plasma não está disponível (PAGE-SHARP et al., 2015).

Amostras de sangue capilar em DBS têm sido utilizadas no diagnóstico de erros inatos do metabolismo e na detecção e quantificação de HIV, vírus da hepatite B e plasmódios em ambientes de pesquisa. A dosagem da concentração de medicamentos em DBS representa um novo método de superar dificuldades como a necessidade de múltiplas coletas de sangue em neonatos e crianças, onde a coleta de grandes volumes de sangue poderá resultar em efeitos hemodinâmicos adversos (PAGE-SHARP et al., 2015).

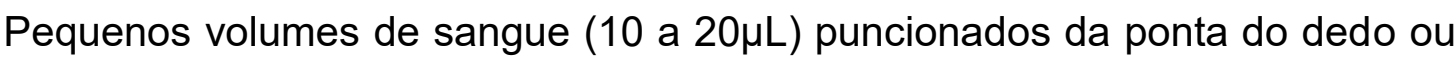
do calcanhar podem ser coletados em série e impregnados em papel filtro, amazenados com um agente dessecante, sem necessidade de centrifugar o sangue total e de congelar as respectivas frações do sangue (PAGE-SHARP et al., 2015).

Apesar das vantagens citadas acima, poucos estudos têm demonstrado validação experimental detalhada em humanos que permita o uso de DBS para dosagem da concentração sanguínea de medicamentos (PAGE-SHARP et al., 2015).

Apesar das inúmeras vantagens da coleta por DBS em relação à punção venosa, algumas desvantagens no uso de DBS devem ser consideradas: a auto coleta não está livre de contaminação, e o uso de dados a partir de DBS no MT requer extensa validação clínica, uma vez que o sangue capilar pode apresentar diferentes concentrações em relação ao sangue venoso. Observa-se, ainda, que a variação do hematócrito - Hct - afeta o volume de sangue fixado no papel filtro (ANTUNES et al., 2016). A Tabela 1 resume as principais vantagens e desvantagens da coleta de DBS. 
Tabela 1 - Vantagens e desvantagens da coleta de DBS comparado à coleta de sangue venoso.

\begin{tabular}{|c|c|}
\hline Vantagens & Desvantagens \\
\hline $\begin{array}{l}\text { - coleta fácil e minimamente invasiva: o } \\
\text { paciente pode fazer a coleta em casa, } \\
\text { sem necessidade de coletador } \\
\text { profissional. Por ser mais conveniente, } \\
\text { facilita para cálculo da área sob a curva } \\
\text { de concentração x tempo. }\end{array}$ & $\begin{array}{c}\text { - a concentração capilar pode ser diferente } \\
\text { da concentração venosa: o sangue em } \\
\text { DBS consiste de células sanguíneas, } \\
\text { plasma e fluido intersticial, e o sangue } \\
\text { venoso é centrifugado e tem as células } \\
\text { sanguíneas separadas do soro/plasma. }\end{array}$ \\
\hline $\begin{array}{l}\text { - é requerido pequeno volume de sangue: } \\
\text { ideal para crianças e neonatos. }\end{array}$ & serva. \\
\hline $\begin{array}{c}\text { - a maioria dos analitos são mais estáveis } \\
\text { em DBS quando comparados com } \\
\text { amostras congeladas. }\end{array}$ & $\begin{array}{c}\text { - se não houver treinamento, a coleta pode } \\
\text { não ser bem-sucedida. }\end{array}$ \\
\hline $\begin{array}{l}\text { - transporte e armazenamento } \\
\text { convenientes: seguro em relação à } \\
\text { contaminação de quem o manipula, pode } \\
\text { ser enviado por sistemas postais } \\
\text { tradicionais sem tratamentos especiais; } \\
\text { todas as proteínas, patógenos e enzimas } \\
\text { são inativadas no papel e o crescimento } \\
\text { bacteriano é prevenido. }\end{array}$ & $\begin{array}{c}\text { - é necessária extensa validação: avaliar o } \\
\text { impacto da variação do hematócrito no } \\
\text { tamanho da mancha e na homogeneidade } \\
\text { da amostra. Avaliar o tipo de papel bem } \\
\text { como diferentes fabricantes. }\end{array}$ \\
\hline $\begin{array}{l}\text { - menos dolorosa: mais conveniente para } \\
\text { neonatos e crianças, bem como pacientes } \\
\text { com flebite. }\end{array}$ & $\begin{array}{l}\text { - por oferecer menor volume de sangue, } \\
\text { requer técnica analítica mais sensível. }\end{array}$ \\
\hline $\begin{array}{c}\text { - reduz o número de passos pré-analíticos: } \\
\text { não requer refrigeração nem } \\
\text { centrifugação }\end{array}$ & \\
\hline
\end{tabular}

Fonte: WILHELM et al., 2014; SHARMA et al., 2014; ENDERLE et al., 2016.

A análise de amostras por DBS, quando comparada com amostras de sangue e plasma, é relativamente nova e há pouca informação disponível na validação do método (WILHELM et al., 2014). Em revisão publicada em 2014 por Wilhelm e col., apenas os antimicrobianos ertapenem, linezolida, metronidazol e moxifloxacin foram testados para o método de DBS. 


\section{Garantia da Qualidade em amostras de DBS}

Para garantir resultados significativos, é essencial um controle de qualidade adequado em testes para o MT. Diversas variáveis pré-analíticas, analíticas e pósanaliticas influenciam a análise e devem ser consideradas ao desenvolver e validar novas técnicas de DBS (ANTUNES et al., 2016).

A escolha da matriz é importante pois influencia na recuperação da amostra, na interação da matriz com o sangue, na estabilidade do analito, na análise cromatográfica, na influência do Hct e no volume de sangue absorvido. Os papéis se diferenciam em sua composição, espessura e resistência ao espalhamento do sangue. A matriz mais comum em análises por DBS é o papel de celulose. Existem dois tipos de cartões de papel disponíveis comercialmente: os quimicamente tratados e os não tratados (ANTUNES et al., 2016; SHARMA et al., 2014; WILHELM et al., 2014).

Os cartões não tratados quimicamente mais comumente utilizados são Whartman $903 \AA$ e Ahlstrom $226 \AA$, ambos registrados no FDA (FOOD AND DRUG ADMINISTRATION, 2001). Os cartões que passam por tratamento químico visam a lise celular, a inativação de patógenos e a desnaturação de enzimas e outras proteínas. Os mais utilizados são Whartman FTA®, FTA Elute®, FTA DMPK-A®, e FTA DMPK-B® (ANTUNES et al., 2016; Shama, 2014; WILHELM et al., 2014). Encontra-se disponível uma matriz de papel cuja base não é celulose, e não é tratada quimicamente. Wilhelm e col. e Antunes e col. citam essa matriz como alternativa aos cartões de celulose pois sofre menos influência do Hct e menor interferência na análise por LC-MS/MS por não conter celulose.

A fim de obter manchas de sangue em papel filtro reprodutíveis, o Forum Europeu de Bioanálises (European Bioanalisys Forum - EBF) estabeleceu, em 2011, as Boas Práticas de Coleta de Mancha de Sangue (Good Blood-Spotting Parctices GBSP) (TIMMERMAN et al., 2011), com atualização em 2013 (TIMMERMAN et al., 2013). As orientações visam padronizar os procedimentos pré-analíticos a fim de evitar potenciais fontes de erro como sobreposição de manchas ou borrões, pouco volume de sangue, aplicação de múltiplas gotas, e contaminação resultante de anestésico tópico, desinfetantes ou manipulação do medicamento antes da coleta (ANTUNES et al., 2016). As recomendações para o procedimento de coleta estão listadas no Quadro 1 e sua ilustração está representada pela Figura 2. 
Quadro 1 - Recomendações para o procedimento de coleta de sangue capilar em manchas de papel (ANTUNES et al., 2016; SHARMA et al., 2014).

1. Evitar qualquer contato com a área de coleta da matriz de papel.

2. Se as mãos do paciente estiverem frias, massagear ou aquecer o sítio da coleta a fim de estimular o fluxo sanguíneo local.

3. Higienizar o sítio da punção com álcool isopropílico $70 \%$.

4. Utilizar lanceta estéril e descartável para puncionar o centro da ponta do dedo médio ou anelar.

5. Descartar a primeira gota de sangue pois contém maior quantidade de fluido tecidual.

6. Posicionar cuidadosamente o papel embaixo do dedo e permitir que a gota caia por gravidade. O dedo do paciente nunca deve encostar no papel.

7. Coletar até 5 gotas (média de $50 \mu \mathrm{L}$ ). Para estimular o fluxo sanguíneo, ordenhar gentilmente a mão a partir do punho até a base do dedo, evitando apertar o dedo. Não sobreponha as gotas para evitar concentração da amostra.

8. Deixar a mancha de sangue secar por 3 a 4 horas em posição horizontal, permitindo que os dois lados da matriz esteja em contato com o ar. Evitar tocar a mancha de sangue e impedir que a mesma entre em contato com superfícies enquanto ainda estiver úmida. Evitar exposição ao sol, poeira, ou insetos pois podem comprometer sua integridade.

9. Armazenar a matriz em sacos plásticos contendo dessecante, a temperatura ambiente, refrigerado ou congelado, dependendo da estabilidade do analito.

Figura 2 - llustração do procedimento de coleta de sangue capilar em manchas de papel. A) Punção digital com lanceta estéril. B) Permitir que a gota de sangue caia sobre o papel, sem encostar o dedo.

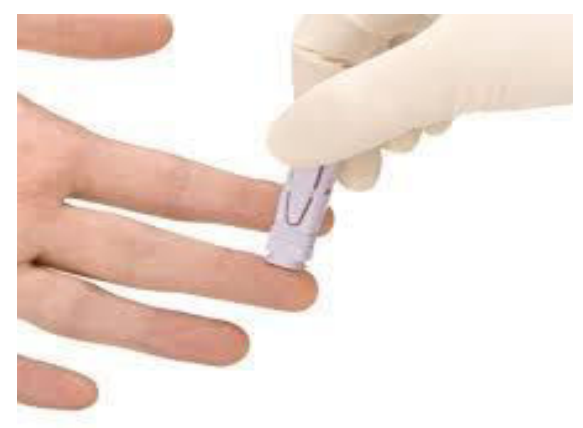

A)

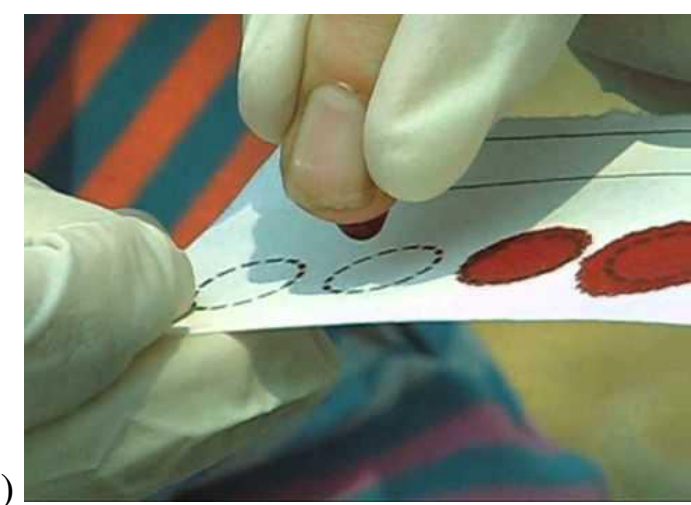




\section{Influência do Hematócrito em amostras de DBS}

Entre os fatores que influenciam na difusão do sangue no papel filtro, o Hct é identificado como o parâmetro mais importante, podendo impactar na validação dos resultados gerados pelo método de DBS. A variação do Hct pode afetar a formação da mancha, o tamanho da mancha, o tempo de secagem, a homogeneidade, a recuperação do analito, a robustez e reprodutibilidade das análises. Existem algumas estratégias a fim de evitar ou minimizar a influência do hematócrito no método de DBS, como analisar a mancha de sangue inteira, eliminando as variações de espalhamento e homogeneidade, e a estratégia mais promissora: medir os níveis de potássio, cujas concentrações estão relacionadas diretamente com o Hct, permitindo a consequente correção dos resultados analíticos (ANTUNES et al., 2016; WILHELM et al., 2014; den BURGER et al., 2015; VU et al., 2011).

\section{Métodos Utilizados para Dosagem de Fármacos em Amostras de DBS}

No laboratório, um disco de diâmetro conhecido é cortado da mancha de sangue do papel, representando um volume fixo do sangue absorvido. Depois da extração, o analito é mensurado com técnica analítica (ENDERLE et al., 2016; WILHELM et al., 2014).

Apesar das vantagens descritas, o uso de DBS é um desafio analítico em função da pequena quantidade de sangue $(5$ - $50 \mu \mathrm{L})$ e da complexidade que amostras de DBS representam. Por isso, a análise de amostras de DBS requer técnicas analíticas sensíveis e caras (ENDERLE et al., 2016; ANTUNES et al., 2016). O custo inicialmente mais elevado em relação às técnicas usualmente utilizadas - como o imunoensaio - torna-se vantajoso no balanço financeiro, pois a otimização da terapia microbiana a partir da coleta por DBS reflete em economia, considerando a redução do tempo de tratamento e antecipação da alta hospitalar.

Devido a sua alta sensibilidade e seletividade, métodos de Espectrometria de Massas (Mass Spectrometry - MS) associados à separação cromatográfica têm sido a ferramenta analítica predominante em análises de DBS (ANTUNES et al., 2016; KEEVIL, 2011). Há aproximadamente 20 anos, a Cromatografia Líquida acoplada à Espectrometria de Massas em "tandem" (LC-MS/MS) começou a ser utilizada na dosagem de analitos a partir de sangue capilar coletado através de gota de sangue seco em papel, ou DBS - Dried Blood Spots. A introdução da LC-MS/MS permitiu o 
desenvolvimento de metodologias de dosagem usando microquantidades de sangue com excelente sensibilidade e precisão (KEEVIL, 2011).

\section{Cromatografia Líquida de Alta Eficiência - HPLC ou CLAE}

A cromatografia líquida de alta eficiência (HPLC ou CLAE) é uma das principais técnicas utilizadas na análise de compostos não voláteis e/ou termicamente instáveis. Está entre as principais técnicas de separação, especialmente em análise de substâncias presentes em matrizes complexas como fluidos biológicos. Apesar de ser excelente quando o objetivo é a separação de compostos, necessita de outra técnica confirmatória quando há necessidade da confirmação da identidade química do analito. Assim, a espectrometria de massas (MS) é a técnica que melhor informa as características estruturais necessárias para sua identificação. O acoplamento entre HPLC e MS combina as vantagens da cromatografia, como a alta seletividade e a eficiência de separação, com as vantagens da espectrometria de massas, como a obtenção de informação estrutural, massa molar e aumento adicional da seletividade. Assim, obtém-se uma ferramenta analítica versátil e de grande potencial para análises qualitativas e quantitativas: a LC/MS - Cromatografia Líquida acoplada à Espectrometria de Massas (CHIARADIA et al., 2008; LANÇAS , 2009).

\section{Espectrometria de Massas - Mass Spectrometry - MS}

A MS é baseada na medida de átomos e moléculas para determinar suas massas moleculares. Para isso, aplica-se carga nas moléculas de interesse e então se mede como a trajetória dos íons resultantes respondem, sob vácuo, a várias combinações de campos elétricos e magnéticos (LANÇAS , 2009).

No espectrômetro de massa (EM), a amostra é introduzida em um "inlet" (dispositivo para introdução da amostra) e direcionada para uma fonte de ionização. No caso do acoplamento da MS com a HPLC, é usado um cromatógrafo líquido como fonte de introdução da amostra no EM. Assim, os picos cromatográficos gerados pela HPLC são individualmente introduzidos na fonte de ionização do EM gerando íons a serem posteriormente separados no analisador e direcionados para a detecção e quantificação. Um software acoplado ao analisador efetua os cálculos e gera os espectros de massas (LANÇAS , 2009). 


\section{Cromatografia Líquida de Alta Eficiência associada à Espectometria de Masas - LC/MS}

O acoplamento entre as técnicas de HPLC e MS - LC/MS - traz benefícios especialmente devido às novas exigências de melhora na sensibilidade dos métodos e à exigência de maiores critérios para identificação de compostos de interesse na área ambiental, saúde, forense, alimentos e outras (LANÇAS , 2009; MCMASTER, 2005).

A Espectrometria de Massas em "tandem" (MS/MS), ou seja, em sequência, é uma técnica espectrométrica que, ao invés de utilizar apenas um analisador de massas para separar os íons gerados na fonte de ionização, utiliza dois estágios de espectrometria de massas, no qual um deles é usado para isolar o íon de interesse e o outro é usado para estabelecer uma relação entre este íon de interesse isolado e outros íons que foram gerados a partir da sua decomposição induzida. Acoplada à cromatografia líquida, a MS/MS, ou seja, a LC-MS/MS é amplamente empregada na detecção de microquantidades de compostos em matrizes complexas, uma vez que possibilita um aumento na detectabilidade e reduz a interferência espectral de compostos presentes na matriz, além de fornecer maior quantidade de informação estrutural (CHIARADIA et al., 2008).

\section{Validação da técnica de coleta de sangue por DBS}

Conforme as diretrizes publicadas pelo FDA e pela Agência Européia de Medicamentos (EUROPEAN MEDICINES AGENCY - EMA), a validação de qualquer método bioanalítico de quantificação de analitos inclui seletividade, acurácia, precisão, recuperação (eficiência da extração), calibração, sensibilidade, reprodutibilidade e estabilidade (FDA, 2013; EMA, 2011). Entretanto, conforme a recomendação do European Bioanalysis Forum (EBF) (TIMMERMAN et al., 2011), a validação do método de DBS requer a análise de parâmetros específicos como a influência do hematócrito, da hemólise e do uso de anticoagulante - se for o caso -, a homogeneidade da amostra, o preparo de amostras de calibração e de controle de qualidade, diluição das amostras e o efeito do tipo de matriz (papel filtro). Segundo o EBF, também devem ser consideradas as Boas Práticas de Coleta, o volume da mancha de sangue e o tamanho do ponto perfurado da mancha de sangue utilizada 
na análise, homogeneidade da mancha de sangue e o uso de múltiplas amostras da mesma mancha (TIMMERMAN et al., 2011).

A validação clínica do método de DBS é essencial para sua implementação, e deve ser realizada comparando seus resultados com as concentrações medidas a partir de amostras de plasma (ENDERLE et al., 2016). As diretrizes publicadas pelo EMA e pelo Instituto de Padronização Clínica e Laboratorial (CLINICAL AND LABORATORY STANDARDS INSTITUTE, 2002), bem como o artigo publicado por Linnet recomenda o uso de pelo menos 40 pares de amostras como base para a análise estatística para comparação de dois métodos, sendo a análise feita por regressão linear (EMA, 2011, CLSI, 2002, LINNET, 1999).

\section{CONCLUSÕES}

Segundo a Organização Mundial da Saúde OMS, a resistência aos antimicrobianos é um problema de saúde pública que ultrapassa fronteiras. A falta de alternativas aos antibióticos comercializados atualmente requer a otimização da terapia, obtida a partir de estudos PK/PD. O MT é realizado a partir da dosagem dos níveis sanguíneos do antimicrobiano em questão, e o procedimento de coleta de sangue do paciente é determinante para que o MT seja viável. Assim, um método que facilite a rotina assistencial e que proporcione maior conforto para o paciente favorece de forma inquestionável a individualização da terapia medicamentosa através do MT. A partir do conhecimento dos níveis séricos do antibiótico, é possível otimizar a dose, visando diminuir o tempo de tratamento e consequentemente o custo da internação hospitalar, reduzir o risco do surgimento de cepas resistentes, bem como proporcionar maior conforto para o paciente.

\section{REFERÊNCIAS}

ANTUNES MV, CHARÃO MF, LINDEN R. Dried blood spots analysis with mass spectrometry: Potentials and pitfalls in therapeutic drug monitoring. Clinical Biochemestry. 2016 Sep;49(13-14):1035-46. 
BARLAM TF, COSGROVE SE, ABBO LM, MACDOUGALL C, SCHUETZ AN, SEPTIMUS EJ, SRINIVASAN A, DELLIT TH, FALCK-YTTER YT, FISHMAN NO, HAMILTON CW, JENKINS TC, LIPSETT PA, MALANI PN, MAY LS, MORAN GJ, NEUHAUSER MM, NEWLAND JG, OHL CA, SAMORE MH, SEO SK, TRIVEDI KK. Executive Summary: Implementing an Antibiotic Stewardship Program: Guidelines by the Infectious Diseases Society of America and the Society for Healthcare Epidemiology of America. Clinical Infectious Disease. 2016 May 15;62(10):1197-202.

BURTON ME, SHAW LM, SCHENTAG JJ, EVANS WE, eds. Applied Pharmacokinetics and Pharmacodynamics. Fourth edn. Philadelphia, PA: Lippincott, Williams \& Wilkins; 2006.

CHIARADIA MC, COLLINS CH, JARDIM ICSF. O estado da arte da cromatografia associada à espectrometria de massas acoplada à espectrometria de massas na análise de compostos tóxicos em alimentos. Química Nova. 2008; 31(3): 623636.

CLINICAL AND LABORATORY STANDARDS INSTITUTE (CLSI). Method Comparison and Bias Estimation Using Patient Samples: Approved Guideline, $2^{\circ}$ ed., 2002.

den BURGER JC, WILHELM AJ, CHAHBOUNI AC, VOS RM, SINJEWEL A, SWART $E L$. Haematocrit corrected analysis of creatinine in dried blood spots through potassium measurement. Analytical and Bioanalytical Chemistry. 2015 Jan; 407(2):621-7.

ENDERLE Y, FOERSTER K, BURHENNE J. Clinical feasibility of dried blood spots: Analytics, validation, and applications. Journal of Pharmaceutical and Biomedical Anaysisl. 2016 Oct 25; 130:231-243.

EUROPEAN MEDICINES AGENCY (EMA). Guideline on bioanalytical method validation. 2011. Último acesso em 29/10/2017:

http://www.ema.europa.eu/docs/en_GB/document_library/Scientific_guideline/2011/0 8/WC500109686.pdf

FOOD AND DRUG ADMINISTRATION (FDA), U.S. Department of Health and Human Services, Center for Drug Evaluation and Research (CDER), Center for Veterinary Medicine (CVM). Guidance for Industry: Bioanalytical Method Validation, 2001. Último acesso em 29/10/2017: http://www.fda.gov/downloads/Drugs/Guidance/ucm070107.pdf

KATO H, HAGIHARA M, HIRAI J, SAKANASHI D, SUEMATSU H, NISHIYAMA N, KOIZUMI Y, YAMAGISHI Y, MATSUURA K, MIKAMO H. Evaluation of Amikacin Pharmacokinetics and Pharmacodynamics for Optimal Initial Dosing Regimen. Drugs in R\&D. 2017 Jan 6.

KEEVIL BG. The analysis of dried blood spot samples using liquid chromatography tandem mass spectrometry. Clinical Biochemestry. 2011 Jan;44(1):110-8. 
LANÇAS F. A cromatografia líquida moderna e a espectrometria de massas: Finalmente "compatíveis"? Scientia Chromatographica. 2013; 5(1):27-46.

LINNET K. Necessary Sample Size for Method Comparison Studies Based on Regression Analysis. Clin Chem. 1999 Jun;45(6 Pt 1):882-94.

MAHMOUDI L, MOHAMMADPOUR AH, NIKNAM R, AHMADI A, MOJTAHEDZDEH $M$. Limited sampling strategy for estimation of amikacin optimal sampling time in critically ill adults. Anaesthesia and Intensive Care. 2014 Mar;42(2):228-33.

MCMASTER M. LC/MS: A Practical User's Guide. Wiley-Interscience, 2005.

MILOSHESKA D, GRABNAR I, VOVK T. Dried blood spots for monitoring and individualization of antiepileptic drug treatment. European Journal Pharmacological Science. 2015 Jul 30; 75:25-39. Epub 2015 Apr 17.

PAGE-SHARP M, NUNN T, SALMAN S, MOORE BR, BATTY KT, DAVIS TM, MANNING L. Validation and Application of a Dried Blood Spot Ceftriaxone Assay. Antimicrobials Agents Chemotherapy. 2015 Oct 5;60(1):14-23.

PARKER SL, SIME FB, ROBERTS JA. Optimizing dosing of antibiotics in critically ill patients. Current Opinion in Infectious Disease. 2015 Dec;28(6):497-504.

RYBAK M, LOMAESTRO B, ROTSCHAFER JC, MOELLERING R JR, CRAIG W, BILLETER M, DALOVISIO JR, LEVINE DP. Therapeutic monitoring of vancomycin in adult patients: a consensus review of the American Society of HealthSystem Pharmacists, the Infectious Diseases Society of America, and the Society of Infectious Diseases Pharmacists. American Journal of Health-System Pharmacy. 2009 Jan 1;66(1):82-98.

SHARMA A, JAISWAL S, SHUKLA M, LAL J. Dried blood spots: concepts, present status, and future perspectives in bioanalysis. Drug Test Anal. 2014 May;6(5):399-414.

TIMMERMAN P, STEVE WHITE S, GLOBIG S ET AL. EBF recommendation on the validation of bioanalytical methods for dried blood spots. Bioanalysis. 2011; 3(14), 1567-1575.

TIMMERMAN P, WHITE S, COBB Z, DE VRIES R, THOMAS E, van BAAR B; European Bioanalysis Forum. Update of the EBF recommendation for the use of DBS in regulated bioanalysis integrating the conclusions from the EBF DBS-microsampling consortium. Bioanalysis. 2013 Sep;5(17):2129-36.

VU DH, KOSTER RA, ALFFENAAR JW, BROUWERS JR, UGES DR. Determination of moxifloxacin in dried blood spots using LC-MS/MS and the impact of the hematocrit and blood volume. Journal of Chromatography. B, Analytical Technologies in the Biomedical and Life Sciences. 2011 May 1;879(15-16):1063-70. 
WILHELM AJ, DEN BURGER JC, SWART EL. Therapeutic drug monitoring by dried blood spot: progress to date and future directions. Clinical Pharmacokinetics. $2014 \mathrm{Nov} ; 53(11): 961-73$. 